Résumés des conférences et travaux

\title{
Philologie moyen-indienne
}

Nalini Balbir

\section{(2) OpenEdition \\ Journals}

Édition électronique

URL : https://journals.openedition.org/ashp/4759

DOI : $10.4000 /$ ashp.4759

ISSN : 1969-6310

Éditeur

Publications de l'École Pratique des Hautes Études

\section{Édition imprimée}

Date de publication : 1 septembre 2021

Pagination : 384-390

ISSN : 0766-0677

Référence électronique

Nalini Balbir, «Philologie moyen-indienne », Annuaire de l'École pratique des hautes études (EPHE), Section des sciences historiques et philologiques [En ligne], 152 | 2021, mis en ligne le 14 juin 2021, consulté le 16 juin 2022. URL : http://journals.openedition.org/ashp/4759 ; DOI : https://doi.org/ 10.4000/ashp.4759 


\title{
PHILOLOGIE MOYEN-INDIENNE
}

\author{
Directrice d'études : $\mathrm{M}^{\mathrm{me}}$ Nalini BALBIR
}

Programme de l'année 2019-2020 : I. Initiation au pali. - II. Examen de travaux récents dans le domaine du moyen-indien et lecture de textes en pali.

L'auditoire de 2019-2020 consistait en étudiants bien formés et avancés aux intérêts variés. Nous avons donc jugé possible de poursuivre l'exploration de la littérature bouddhique en prakrit gāndhārī amorcée en 2018-2019, toujours en prenant pour base le pali ainsi qu'en procédant à des comparaisons avec les parallèles en sanskrit bouddhique, et, le cas échéant, en d'autres langues. La synthèse magistrale de R. Salomon (2018) est à la fois un appui et une source excellente de textes bien choisis.

Nous avons commencé l'année par la lecture du Bhikkhuvagga figurant dans le Dhammapada en pali (chap. 25), attirant l'attention en particulier sur les procédés rhétoriques à l'œuvre dans ce chapitre qui fait usage de jeux de langage (récurrence délibérée du chiffre " cinq » en 370) et d'un refrain définissant « le vrai moine » (pérennité d'une formule que l'on retrouve également dans deux leçons du canon jaina, cf. Alsdorf 1974 [1962] : p. 230-243, même si les contenus eux-mêmes ne sont pas superposables). Nous avons examiné de près les termes difficiles tels que mantabhānin (Dhp 363), pour lequel nous sommes parvenus à des conclusions que confirme l'article sous presse de Martin Straube (voir références ci-dessous). Bien que plusieurs savants aient proposé de faire correspondre manta à manda (en dernier Oberlies 2019, que nous avons présenté au cours des séances), cette hypothèse est peu convaincante, en particulier parce que manda a généralement des connotations négatives, qui sont en contradiction avec ce que veut exprimer la séquence récurrente mantabhān̄i anuddhato laquelle, au contraire, est très positive. À partir de là, il est préférable de relier manta à manta, de prendre au sérieux les équivalences avec pañ̃̃a « intelligence » données par les commentaires et d'aller plus loin encore dans la démonstration sur la base des parallèles :

To sum up, in view of the evidence gathered together-scil., the semantic overlap between Pali mantā and mantabhānin which is also acknowledged by the commentaries, the testimony of the PDhp, and the Gāndhārī commentary on the Sanigitisūtra-I would suggest to derive manta- in Pali mantabhānin from the absolutive mantā, and understand the whole expression to mean "speaking with/after reflection" (Straube sous presse).

La lecture du chapitre a été comparative, renvoyant à l'Udānavarga en sanskrit ainsi qu'au Dharmapada de Patna (PDhp, cf. Cone 1989). En outre, pour accoutumer l'auditoire au style des commentaires palis, nous avons également étudié, à titre d'exemple, le commentaire relatif aux strophes 369-373. En plus des gloses, le passage élabore les termes techniques des strophes en fonction des grilles abhidharmiques. Le pāda formulaire final abbhā mutto va candimā « comme la lune libérée du nuage » (382) a été l'occasion de faire un point sur les dérivés moyen-indiens en -imā, 
et notamment sur la forme palie du nom de la lune (Caillat 1975). Cette exploration a été poursuivie par l'examen du sutta afférent dans la Saddaniti, grammaire palie du moine birman Aggavaṃsa (XII ${ }^{\mathrm{e}}$ s.; éd. H. Smith, § 794, p. 793) qui défend candimā comme seule forme à admettre en pali, contre candamā qui est influencée par le sanskrit et, dit-il, n'est donc pas appropriée (abbhā mutto va candimā ti hi pā l̄i dissati, keci pana sakkațabhāsato nayam gahetvā candamā ti paṭanti, tam na yuttam). C'est un exemple parmi d'autres de l'attitude très claire de ce grammairien, unique en son genre, qui, partisan d'un purisme pali et soucieux d'établir une norme grammaticale typique de cette langue, cherche à en éliminer ce qui trahit la présence du sanskrit. Cette position est certainement liée au contexte birman de son époque où la tradition des lettrés sanskrits joue un rôle croissant. Munis d'une bonne compréhension de la version en pali du Bhikkhuvagga, nous nous sommes attaqués à la version en gāndhārī (chap. II, strophes 51-90) éditée par John Brough (1962). Les discussions relatives à l'interprétation des détails philologiques ont été nombreuses. L'un des mots problématiques pour la compréhension duquel Brough a montré l'utilité d'un parallèle tokharien est discuté ci-dessous par Timothée Chamot-Rooke, auditeur de la conférence, à qui on avait demandé de reprendre le dossier. En outre, il se trouve que cette section particulière du Dharmapada en gāndhārī est connue par un autre manuscrit (Lenz 2003). Cette situation est tout à fait unique, car les chercheurs sont généralement déjà fort satisfaits d'avoir à leur disposition un seul manuscrit en cette langue pour un texte donné (Lenz 2003, p. 14 et suiv.). On a tenté de tirer le meilleur parti de ce que permet cette comparaison, déjà fort détaillée dans le livre de Lenz.

Enfin, plus largement, le chapitre Bhikkhu de la mouvance Dharmapada s'attache à définir les frontières entre moine et maître de maison. Cette dernière notion ayant donné lieu à un riche ouvrage collectif (Olivelle éd. 2019) appelé à devenir incontournable, nous en avons brièvement porté le contenu à la connaissance du public de la conférence.

La littérature narrative en gāndhārī sous la forme la plus élémentaire du pürvayoga, qui fournit un canevas plus qu'un véritable récit et représente une forme à la frontière entre l'oral et l'écrit (dont on a des équivalents dans les histoires en style télégraphique qui parcourent les premiers commentaires en prose jaina, les cūrnii), nous a ensuite occupés. Nous l'avons abordée d'une part avec l'histoire de Sudașa, qui, en quelques phrases, résume les thèmes principaux connus par l'histoire du prince Vessantara, modèle de générosité (Lenz 2003 : p. 157) et d'autre part avec celle du Bodhisattva marchand de mer. Cette dernière (Lenz 2003 : p. 150, pūrvayoga I), dont on a lu en parallèle la version sanskrite transmise par le Sanghabhedavastu (sanskrit bouddhique, éd. Gnoli 1978 : p. 13-14 «The story of the merchant Dhanaratha », donnée en appendice par Lenz 2003, mais dont il est préférable de consulter l'original), est d'une brièveté déconcertante. L'analyse fouillée de T. Lenz doit être complétée par la discussion de ce récit de vie antérieure figurant dans le compte rendu de von Hinüber 2004.

Nous allions continuer l'exploration de la littérature narrative en gāndhārī à travers l'étude de poèmes contenus dans l'Anavatapta-gāthā en retenant celui consacré à Sumanas que nous voulions pour commencer aborder dans sa version sanskrite, lorsque la France est entrée en confinement. Un certain état de sidération et, à l'époque, le manque de familiarité avec les possibilités offertes par la visioconférence se sont 
conjugués pour entraîner, dans notre cas, l'annulation pure et simple des séances, ainsi que l'annulation de la tenue de la Quatrième semaine internationale d'études palies prévue pour juin 2020 (avec le soutien des actions ponctuelles de l'EPHE). Durant les mois de printemps, nous avons donc privilégié un suivi individuel des étudiants, qui ont remis les travaux nécessaires à la validation du séminaire puis ont poursuivi leurs recherches personnelles sans faillir. Ainsi, M. Victor Baptiste a complété un excellent mémoire de master 1 (dans le domaine des études indo-persanes, voir activités de la directrice d'études) puis présenté sa candidature au programme gradué en sciences des religions pour une bourse d'excellence de master 2, qu'il a obtenue. D'autre part, entre avril et juin, le directeur d'études a révisé plusieurs des travaux en cours de M. Javier Schnake (docteur EPHE 2018, boursier de la Pali Text Society et du Lumbini International Research Institute) : son édition critique du Vajirasāratthasañgaha précédée d'une introduction en anglais (sous presse à la Pali Text Society), l'édition et la traduction de l'Aḍ̣habhāganidāna, version en pali, pour un article qu'il prépare en collaboration avec M. Lorrillard (pour la partie lao), ainsi que l'édition et la traduction du Sotatthakimahānidāna qu'il a remises à l'éditeur à l'automne 2020 pour publication à Bangkok.

\section{Références bibliographiques}

Ludwig Alsdorf, « Uttarajjhāyā Studies », Indo-Iranian Journal, 6 (1962), p. 110-136 = Kleine Schriften, éd. A. Wezler, Wiesbaden, F. Steiner Verlag, 1974, p. 230-243.

John Brough, The Gāndhāri Dharmapada, Londres, Oxford UP, 1962.

Colette Caillat, "À propos de sanskrit candrimā "clair de lune" ", dans Mélanges linguistiques offerts à Émile Benveniste, Louvain, Peeters, 1975, p. 65-74; reproduit dans C. Caillat, Selected Papers, N. Balbir, O. von Hinüber (éd.), Bristol, Pali Text Society, 2011.

Margaret Cone, « Patna Dharmapada I », Journal of the Pali Text Society, 13 (1989), p. 101-218.

Raniero Gnoli, The Gilgit Manuscript of the Sanghabhedavastu. Being the 17th and Last Section of the Vinaya of the Mūlasarvāstivādin, II, R. Gnoli, T. Venkatacharya (éd.), Rome, Istituto Italiano per il medio ed estremo oriente, 1978.

Timothy Lenz, A New Version of the Gāndhārī Dharmapada and a Collection of Previous-Birth Stories, Seattle, University of Washington Press, 2003.

Timothy Lenz, « Once Upon a Time - An Avadānist from Gandhāra », Buddhist Studies Review 21, 2 (2004), p. 197-215.

Thomas Oberlies, Pāli Grammar: The Language of the Canonical Texts of Theravāda Buddhism, vol. I. Phonology and Morphology, Bristol, Pali Text Society, 2019.

Patrick Olivelle (éd.), Gṛhastha. The Householder in Ancient Indian Religious Culture, Oxford, Oxford University Press, 2019.

Richard Salomon, Two Gāndhārī Manuscripts of the Songs of Lake Anavatapta (Anavataptagāthā), Seattle, University of Washington Press, 2008.

Richard Salomon, The Buddhist Literature of Ancient Gandhära. An Introduction with Selected Translations, Somerville (MA), Wisdom Publication, 2018.

Martin Straube, «Pali mantā and mantabhānin », Journal of the Pali Text Society, 34 (2020) sous presse.

Oskar Von Hinüber, Compte rendu de T. Lenz (2003) dans Journal of the American Oriental Society, 124, 4 (2004), p. 803-805.

Site internet : www.gandhari.org 
Appendice : " Note sur GDhp 67 et ses parallèles » par Timothée Chamot-Rooke

(auditeur de la conférence en 2019-2020; doctorant EPHE dans le cadre du projet ERC HisTochText sous la direction de M. Georges-Jean Pinault)

La strophe 67 du Dhammapada en gāndhārī se présente comme suit dans l'édition de John Brough : na bhikhu tavada bhodi | yavada bhikșadi para | veśma dharma samada'i | bhikhu bhodi na tavada \| (Brough 1962 : 128). La traduction qu'en donne Richard Salomon dans The Buddhist Literature of Ancient Gandhara est la suivante : " One does not become a monk merely by begging from others; as long as he lives a domestic life, he is no monk. » (Salomon 2018 : 194). La strophe renvoie dos à dos les religieux errants qui se contentent de mendier sans s'adonner à la doctrine (sk. parivrājaka) et les maîtres de maison (sk. grhastha) ${ }^{1}$. Outre le parallèle du Dhammapada pāli (Dhp 266), on possède pour cette strophe deux parallèles sanskrits (Ud 32.18 et Mv III 422,13*) ainsi qu'un parallèle bilingue sanskrit-tokharien A (A 360). Le parallèle du Dhammapada pāli a un texte un peu différent de la version en gāndhārī : na tena bhikkhu hoti | yāvatā bhikkhate pare | vissam dhammam samādāya | bhikkhu hoti na tāvatā || (Dhp 266, texte cité d'après le site GRETIL). L'hypothèse désormais admise pour expliquer pā. vissam (acc. sg. ou pl.) est de voir dans ce mot le correspondant exact au point de vue formel et sémantique de sk. veśman-, gdh. veśma « maison » selon le développement régulier en pāli de $\breve{e}(<e$ par abrègement) en $i$ devant une palatale en syllabe fermée : sk. veśma(n)- > pā. *věśśs- > vissa-, cf. pā. issāmi « j'irai », ānissāmi « j'apporterai », pațivissaka- « voisin » en face de sk. eșyāmi, āneșyāmi, prativeśya[ka]- (Oberlies 2019 : 81-82, Norman 1997 : 152). Cette lecture de pā. vissam au sens de «maison»s'appuie également sur le texte sanskrit, tel qu'édité par Franz Bernhard (Bernhard 1965: 436), de la strophe correspondante de l'Udānavarga (32.18c : veśmām dharmām samādāya), ainsi que sur le texte sanskrit du fragment bilingue sanskrit-tokharien A 360, qui présente un texte presque identique (veśma-dharmām samādhāya) ${ }^{2}$.

Le fragment bilingue sanskrit-tokharien (A 360) est l'un des sept fragments (A 359-A 365) d'un manuscrit qui nous est parvenu dans un état lacunaire et qui constitue une sorte d'anthologie de textes bouddhistes plus ou moins longs, glosés par une traduction en tokharien A (sur A 360-361, voir récemment Weiss 2017: 4 et suiv.). La strophe sanskrite de ce fragment a été identifiée par Sieg et Siegling (TS : 201) comme parallèle aux strophes citées de l'Udānavarga et du Dhammapada (sur la réception de l'Udānavarga dans la région où les langues tokhariennes étaient parlées, voir Peyrot 2016, avec références antérieures). Elle figure isolée entre deux textes de longueur plus importante. Nous en reproduisons ici le texte d'après le site СЕтом :

11 /// penu näṣ | 5 10-5 suträ | bhikṣur na tāvatā bhavati | ṣāmam mā täppreṃ ṣolār mäskaträ ///

1. Sur cette double opposition dans le bouddhisme ancien, voir Chakravarti 1983.

2. Le Mahāvastu (III 422,13*) a un texte différent : viṣamāim dharmāim samādāya. L'adjectif sk. vișama« irrégulier » est issu d'une rétro-formation fautive à partir de la variante dialectale visama citée dans le commentaire pāli au Dhammapada à côté de pā. vissa «maison » (Norman 1997 : p. 152). 
12 /// pā(n)äșträ ālyeksäṣ | veśma-dharmām samādhāya | waṣtașiṃ wkäṃ etsu(räṣ) /// 13 /// täppreṃ șolār tākiṣ | 10-6 suträ || yugam vā | mu ///

La strophe et sa traduction en prose, pāda par pāda ${ }^{4}$, sont introduites à la ligne 11 par le mot suträ et le chiffre 15. Le texte sanskrit des pāda b et d ainsi que le début de leur traduction en tokharien ont disparu dans la lacune. À la fin de la ligne 13 (dernière ligne du fragment), les mots yugam vā appartiennent au début d'un autre texte (voir Weiss 2017 : 4). Le composé sanskrit veśma-dharmām (acc. pl.), que l'on peut traduire par « devoirs relatifs à la maison, responsabilités du maître de maison » ${ }^{5}$, est rendu par tokharien A waștașiṇ wkäm « mode de vie relatif à la maison », selon le procédé, courant en tokharien, qui consiste à traduire le premier membre d'un composé sanskrit tatpuruṣa par un adjectif relationnel en -și, cf. nada-āgāra- " maison de roseaux » = kärwāṣi wașt (A 354 b6), dharma-vinaya- "discipline de la loi » = märkampalși enäșlune (A 354 b6), jāti-samnsāra- "cercle des (re)naissances »= cmolwāṣim sark (A 354 b6), etc. On peut donc raisonnablement supposer, comme le fait K. R. Norman (Norman 1997 : 152), que le texte en gāndhārī présente le même composé veśma-dharma (à l'acc. pl.), ce qui, d'un point de vue sémantique et syntaxique, est bien plus satisfaisant que d'y lire deux mots indépendants.

La traduction tokharienne du pāda $d$ suppose un texte sanskrit légèrement différent de celui qui nous est transmis par la recension de Bernhard de l'Udānavarga. L'optatif tokharien P3 sg. tākis (de la racine nas- « être ») ne peut traduire que sanskrit bhavet ${ }^{6}$, alors qu'au même pāda l'Udānavarga de Bernhard a le présent bhavati, comme au

3. Noter la différence avec la racine employée dans 1'Udānavarga de Bernhard. Dans les textes en sanskrit bouddhique, la racine $s a m-\bar{a}-d \bar{a}$ - signifie « adopter, prendre sur soi », en parlant d'une obligation morale ou religieuse (BHSD : 568b; Stefan Baums et Andrew Glass traduisent gā. samadadi par « takes up » dans leur Dictionary of Gāndhār $\bar{l}$ ); elle est très souvent employée à l'absolutif et précédée d'une forme conjuguée de la racine vrt- au sens de «mettre en pratique ». On la recontre par exemple avec dharmān (acc. pl.) pour objet dans le Mahāparinirvāṇasūtra, dans l'expression récurrente imān [...] dharmān samādāya vartisyante " ayant adopté ces (bons) dharmas, ils les mettront en pratique » (MPNS $1.38,2.13,2.28,2.36$ ). La racine $s a m-\bar{a}-d h \bar{a}$-, quant à elle, n'appartient pas spécifiquement à la phraséologie bouddhique; elle s'emploie notamment au sens d' «endosser, enfiler» un vêtement, mais aussi «s'adonner, se consacrer à » (+ acc.). Le sens des deux racines est proche, et elles ont pu être confondues. En TA, l'expression wkäm entsa- signifie «prendre, adopter, embrasser le mode de vie », cf. YQ II.14 a2-3 pukis ārtunt wkäm näs penu em (tsäsmār) « I, too, am going to embrace the way revered by everyone » (= « the way of monkhood», a 2 șāmnuneșin wkäm) [trad. СЕтом].

4. Ici, la traduction tokharienne n'a que valeur de glose, ce qui est conforme à l'affirmation de M. Peyrot selon laquelle « speakers of Tokharien preferred the Udānavarga in the Sanskrit original to a translation into their native language. » (Peyrot $2016:$ p. 324).

5. Dans la traduction chinoise du Mahāparinirvānasūtra, il est aussi question de nonnes qui, en faveur du dharma religieux, « dénigrent le dharma domestique » (« disparage the domestic dharma » selon la traduction de Mark L. Blum (Blum 2013 : p. 10). Blum précise en note le sens de cette expression : « In other words, they complained openly about what was expected of them as homemakers. There are many kinds of dharmas from many different sources presented in the MPNS, with the Buddha's dharma usually identified as such. In this particular case, the allusion is to dharma in the sense of social duty. » (Blum 2013 : p. 340).

6. Pour la traduction de l'optatif sanskrit par un optatif en tokharien, voir par exemple A 461 b2 : vivecayet • lyutkāṣiṣ. On pourrait objecter qu'un contre-exemple se trouve en 387 a4 : tat kiṃ-niśrtaṃ bhavatīti • säm kuc-semum «tä» tākiṣ tanne wkäñ (yo) « celui-ci, sur quoi repose-t-il? », où sk. bhavati est glosé par l'optatif tākiṣ. Mais dans notre texte, on ne voit pas bien pourquoi le traducteur tokharien aurait choisi de traduire bhavati par mäskaträ dans le pāda $a$ et par tākiṣ dans le pāda $d$ si le texte 
pāda $a$ : bhikșur na tāvatā bhavati | yāvatā bhikșate parān | veśmām dharmām samādāya | bhikșur bhavati na tāvatā \| (Ud 32.18). La traduction tokharienne, qui suit scrupuleusement l'ordre des mots du sanskrit, suppose en outre un ordre des mots différent de celui de l'Udānavarga tel qu'édité par Bernhard. La strophe bilingue du fragment A 360 peut donc, sur la base des parallèles cités plus haut et de la traduction tokharienne, être restituée de la manière suivante :

a bhikṣur na tāvatā bhavati | șāmaṃ mā täppreṃ ṣolār mäskaträ (|

b yāvatā bhikșate parān | kos-ne ${ }^{7}$ ) pā(n)äșträ ālyeksäṣ |

c veśma-dharmām samādhāya | waṣtașim wkäṃ etsu(räṣ |

d bhikṣur na tāvatā bhavet | șāmaṃ mā) täpprem șolār tākiṣ

On n'est pas moine dans la mesure

où on réclame l'aumône auprès d'autrui ;

ayant endossé les responsabilités de [maître de] maison,

dans cette mesure on ne saurait être moine.

Au pāda $d$, la valeur d'impossibilité exprimée par la négation suivie de l'optatif bhavet est cohérente avec l'absolutif du pāda $c$, qui a presque une valeur conditionnelle ici : «si on endosse..., on ne peut pas...». De plus, cette restitution présente pour le quatrième pāda un texte sanskrit plus satisfaisant au plan métrique que celui de l'Udānavarga tel qu'édité par Bernhard ${ }^{8}$. Tout se passe comme si les deux versions sanskrites « du nord » représentaient deux sanskritisations indépendantes plus ou moins fidèles de la strophe en gāndhārī :

\begin{tabular}{|l|l|l|}
\hline Gāndhārī Dh. 67 & Udānavarga 32.18 & A 360 (Sanskrit) \\
\hline na bhikhu tavada bhodi & bhikșur na tāvatā bhavati & bhikșur na tāvatā bhavati \\
yavada bhikșadi para $\mid$ & yāvatā bhikșate parān $\mid$ & (yāvatā bhikṣate parān |) \\
veśma-dharma samada'i & veśmāṃ dharmāṃ samādāya & veśma-dharmāṃ samādhāya \\
bhikhu bhodi na tavada. $\|$ & bhikșur bhavati na tāvatā $\|$ & (bhikșur na tāvatā bhavet $\|$ ) \\
\hline
\end{tabular}

\section{Références bibliographiques}

Baums, S. et Glass, A., A Dictionary of Gāndhārī. http://gandhari.org/dictionary, 2002-

Bernhard, F., Udānavarga. Band I: Einleitung, Beschreibung der Handschriften, Textausgabe, Bibliographie, Göttingen, Vandenhoeck \& Ruprecht, 1965.

sanskrit ne comportait pas cette nuance, d'autant plus que la traduction tokharienne, en prose, n'est sujette à aucune contrainte métrique.

7. Klaus T. Schmidt restitue ici kosprem-ne d'après Couvreur (Schmidt 1974 : p. 147). L'expression täprem... kosprem-ne signifie « aussi souvent... que » (« wie oft » selon SSS : p. 186, § 314). Toutefois le sens de $\operatorname{ta} v a t(\bar{a})$... yāvat( $\bar{a})$ serait mieux rendu par täprem... kos-ne « autant... que », cf. A 213 a2 où sk. yāvad vyāmena est rendu par kos-ne kașyo (SSS : ibid.). Les deux constructions täprem... kos-ne et täprem... kosprem-ne sont bien attestées (voir SSS : ibid.).

8. Le procédé de versification qui consiste, dans un śloka, à employer l'optatif bhavet en fin de pāda pair et le présent bhavati en fin de pāda impair est très courant dans l'Udānavarga, l'inverse étant exclu. Ainsi placée en fin de pāda impair, la forme bhavati induit presque toujours un pāda hypermétrique (U — UU X, à lire $\mathrm{U}-\mathrm{X}$ ), ce qui prouve qu'elle recouvre une forme sanskrite hybride contractée bhoti (BHSG : 28a), attestée par ailleurs sporadiquement dans l'Udānavarga de Subaši (voir par exemple 6c, 59d, 274a dans l'édition de Nakatani 1987; malheureusement la strophe qui nous intéresse est perdue). 
BHSD $=$ Edgerton, F., Buddhist Hybrid Sanskrit Grammar and Dictionary, vol. 2. Dictionary, réimpr. Delhi, Motilal Banarsidass, New Haven (CT), Yale UP, 1953.

BHSG $=$ Edgerton, F., Buddhist Hybrid Sanskrit Grammar and Dictionary, vol. 1. Grammar, réimpr. Delhi, Motilal Banarsidass, New Haven (CT), Yale UP, 1953.

Blum, M. L., The Nirvana Sutra (Mahāparinirvāṇa-sūtra), vol. I, Berkeley (CA), Bukkyō Dendō Kyōkai America, 2013 (BDK English Tripițaka Series).

Brough, J., The Gāndhārī Dharmapada. Edited with an introduction and commentary by John Brough, Londres, Oxford UP, 1962 (London Oriental Series, Volume 7).

Cетом $=$ A Comprehensive Edition of Tocharian Manuscripts. http://www.univie.ac.at/ tocharian/.

Chakravarti, U., « Renouncer and Householder in Early Buddhism », Social Analysis: The International Journal of Social and Cultural Practice, 13 (1983), p. 70-83.

Nakatani, H. (éd.), Udānavarga de Subaši. Édition critique du manuscrit sanskrit sur bois provenant de Subaši, t. I. Texte et fac-similés, Paris, Collège de France, 1987 (Publications de l'Institut de civilisation indienne, 53).

Norman, K. R., A philological approach to Buddhism. The Bukkyō Dendō Kyōkai Lectures 1994, Londres, School of Oriental and African Studies, 1997 (The Buddhist Forum, Vol. V).

Oberlies, T., Päli Grammar: The language of the canonical texts of Theraväda buddhism, vol. I. Phonology and Morphology, Bristol, Pali Text Society, 2019.

Peyrot, M., "The Sanskrit Udānavarga and the Tocharian B Udānastotra: a window on the relationship between religious and popular language on the northern Silk Road », Bulletin of the School of Oriental and African Studies, 79, 2 (2016), p. 305-327.

Salomon, R., The Buddhist Literature of Ancient Gandhära. An Introduction with Selected Translations, Somerville (MA), Wisdom Publications, 2018.

Schmidt, K. T., Die Gebrauchsweisen des Mediums im Tocharischen, thèse, Georg-AugustUniversität zu Göttingen, 1974.

SSS = Sieg, E., Siegling, W. et Schulze, W., Tocharische Grammatik, Göttingen, Vandenhoeck \& Ruprecht, 1931.

TS $=$ Sieg, E. et Siegling, W., Tocharische Sprachreste. [Sprache A.], vol. I. Die Texte, A. Transcription, B. Tafeln, Berlin, Leipzig, Walter de Gruyter, 1921.

Weiss, M., « Le proto-tokharien : langue de non-paysans ? », 36th East Coast Indo-European Conference (ECIEC), Ithaca (NY), 2017. 